\title{
The biological effects of microencapsulated organic acids and botanicals induces tissue- specific and dose-dependent changes to the Gallus gallus microbiota
}

\author{
Kristina M. Feye ${ }^{1}$, Christina L. Swaggerty ${ }^{1^{*}}$ (D) Michael H. Kogut ${ }^{1}$, Steven C. Ricke ${ }^{2}$, Andrea Piva ${ }^{3,4}$ and Ester Grilli ${ }^{3,5}$
}

\begin{abstract}
Background: Microencapsulated organic acids and botanicals have the potential to develop into important tools for the poultry industry. A blend of organic acids and botanicals (AviPlus ${ }^{\oplus} \mathrm{P}$ ) has previously shown to reduce Salmonella and Campylobacter in chickens; however, changes to the microbiota of the jejunum and ileum have not been evaluated. Microbiota diversity is linked to, but not correlated with, the efficacy of natural products; therefore, understanding the effects on the microbiota is necessary for evaluating their potential as an antibiotic alternative.

Results: lleal and jejunal segments from control and supplement-fed chickens (300 and $500 \mathrm{~g} / \mathrm{metric}$ ton [MT]) were subjected to alpha diversity analysis including Shannon's diversity and Pielou's Evenness. In both analytics, the diversity in the ileum was significantly decreased compared to the jejunum irrespective of treatment. Similarly, beta diversity metrics including Bray-Curtis dissimilarity index and Weighted Unifrac Distance Matrix, were significant $(\mathrm{Q}<0.05)$ for both tissue and treatments comparisons. Alpha and beta diversity analytics indicated compartmentalization effects between the ileum and jejunum. Additionally, analysis of communities in the microbiota (ANCOM) analysis showed Lactobacilliaceae predominated the total operational taxonomic units (OTU), with a stepwise increase from $53 \%$ in the no treatment control (NTC) to $56 \%$ in the $300 \mathrm{~g} / \mathrm{MT}$ and $67 \%$ in the $500 \mathrm{~g} / \mathrm{MT}$ group. Staphylococcaceae were $2 \%$ in NTC and 2 and $0 \%$ in 300 and $500 \mathrm{~g} / \mathrm{MT}$ groups. Enterobacteriaceae decreased in the $500 \mathrm{~g} / \mathrm{MT}$ (31\%) and increased in the $300 \mathrm{~g} / \mathrm{MT}$ (37\%) compared to the NTC (35\%). Aerococcaceae was $0 \%$ for both doses and $7 \%$ in NTC. Ruminococcaceae were $0 \%$ in NTC and 2 and $1 \%$ in the 300 and $500 \mathrm{~g} / \mathrm{MT}$. These changes in the microbial consortia were statistically $(Q<0.05)$ associated with treatment groups in the jejunum that were not observed in the ileum. Least discriminant analysis effect size (LEFSE) indicated different changes directly corresponding to treatment. Enterobacteriaceae demonstrated a stepwise decrease (from NTC onward) while Clostridiaceae, were significantly increased in the $500 \mathrm{~g} / \mathrm{MT}$ compared to NTC and $300 \mathrm{~g} / \mathrm{MT}(P<0.05)$.

Conclusion: The bioactive site for the microencapsulated blend of organic acids and botanicals was the jejunum, and dietary inclusion enhanced the GIT microbiota and may be a viable antibiotic alternative for the poultry industry.
\end{abstract}

Keywords: Botanicals, lleum, Jejunum, Microbiota, Microencapsulated, Organic acids

\footnotetext{
* Correspondence: christi.swaggerty@usda.gov

'U.S. Department of Agriculture, Agricultural Research Service, Southern

Plains Agricultural Research Service, $2881 \mathrm{~F}$ and B Road, College Station, TX

77845, USA

Full list of author information is available at the end of the article
}

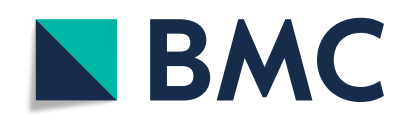

(c) The Author(s). 2020 Open Access This article is licensed under a Creative Commons Attribution 4.0 International License, which permits use, sharing, adaptation, distribution and reproduction in any medium or format, as long as you give appropriate credit to the original author(s) and the source, provide a link to the Creative Commons licence, and indicate if changes were made. The images or other third party material in this article are included in the article's Creative Commons licence, unless indicated otherwise in a credit line to the material. If material is not included in the article's Creative Commons licence and your intended use is not permitted by statutory regulation or exceeds the permitted use, you will need to obtain permission directly from the copyright holder. To view a copy of this licence, visit http://creativecommons.org/licenses/by/4.0/. The Creative Commons Public Domain Dedication waiver (http://creativecommons.org/publicdomain/zero/1.0/) applies to the data made available in this article, unless otherwise stated in a credit line to the data. 


\section{Background}

The public concerns associated with the use of antibiotics in poultry production, and animal agriculture in general, necessitates research into acceptable natural alternatives that promote feed efficiency and food animal health while reducing the burden of foodborne disease. Stepping back from the refined pharmacological fungal metabolites traditionally used in animal agriculture, plant secondary metabolites and essential oils are an attractive avenue of development for use by the poultry industry [1]. Research indicates bioactive natural compounds can decrease the microbial burden on the immune system and promote feed efficiency by improving digestibility and gastrointestinal (GIT) morphology [2-4] and intestinal mucosal barrier function [5] in poultry. Additionally, essential oils and other natural compounds are generally regarded as safe and can be multi-modal in their activation effects including antimicrobial, anti-parasitic, therapeutic, antiinflammatory, and chemotherapeutic properties [1, 6, 7]. There are numerous recent reviews highlighting natural compounds for their potential to serve as antibiotic alternatives including, but not limited to, cinnamon [8], oregano [9], organic acids [10,11] and others [12].

The GIT microbiota actively participates in homeostatic function, nutrient digestion, and biotransformation of compounds. The symbiotic relationship between host commensal microorganisms and the immune system facilitate immune tolerance and development and can have peripheral consequences to overall health and food animal feed efficiency [13-16]. Additionally, as the microbiota directly interacts with feed matrices, natural compounds must not adversely impact the microbiota community structure and stability. Sufficient evidentiary support must therefore demonstrate that the microbiota does not render the natural compounds inert nor that the biotransformation results in bactericidal effects that reduce diversity that corresponds with decreased absorption of nutrients and compounds [17-19].

Compartmentalization, or localization to a particular section the GIT, is important, though often overlooked in poultry feed amendment studies [20]. The activity of natural compounds should result in changes to the compartment of activity which would provide knowledge related to the changes within the microbial community structure and may ultimately provide insight into the biology driving these effects. As microencapsulation technology continues to evolve, the targeted delivery of natural compounds through the harsh environment of the crop to their intended location further down the GIT may serve to improve biological activity [21]. Additionally, a study by Grilli and colleagues showed that microencapsulation allows for the slow release of organic acids in the small intestine of broilers [22].
There are numerous poultry-specific studies in the literature that evaluate the role of essential oils and other natural products. For example, thymol has been shown to be anti-inflammatory with the ability to modulate the microbiota [23], reduce the effects necrotic enteritis [24], and vanillin exhibits antibiotic-like effects [25, 26]. Organic acids also show promise as feed amendments in poultry [11]. Dietary supplementation with benzoic acid influences gut microbial populations [27], and the addition of organic acids and essential oils improves performance and increases disease resistance [10], while propionic and formic acid supplementation improves carcass traits [28].

Clearly, individual feed additive components have been studied; however, the combinatorial effects of the microencapsulated blend of organic acids and botanicals used in the current study remains to be understood. In a previous study by Mohammadi Gheisar et al., broilers fed with microencapsulated organic acids and botanicals had improved performance and feed efficiency as well as an increase in Lactobacillus counts in the feces [29]. As changes in the intestinal microbiota can be one of the drivers to growth performance, one of the objectives of this study was to investigate the impact of microencapsulated organic acid and botanicals on the microbiota. Moreover, our laboratory recently performed a kinome analysis of ileal and jejunal segments collected from broilers on the microencapsulated diet and showed key differences in immune and metabolic signaling pathways compared to controls indicating tissue-specific differences that are directly attributed to the amended diet [30]. Therefore, the other objective of the present study was to evaluate the potential compartmentalized effects of the microencapsulated blend of organic acids and botanicals on the ileum and jejunum populations. It is important to conduct feed additive studies in vivo; therefore, the commercial broiler by-product chickens used in this study were selected as they are representative birds used in today's poultry production. By evaluating community structure and composition, it will be possible to determine if there are any effects on the microbiota due to bioactivity of organic acids and botanicals in specific compartments of the GIT.

\section{Results}

Animal health, well-being, and chick weights

Chickens were monitored daily and no mortality, behavioral changes, or other animal welfare concerns were observed during the course of the study for the controls or those on the supplemented diets. Other than the dietary supplement that included the microencapsulated blend of organic acids and botanicals, the chicks were not administered any medications or other therapeutic interventions during the study. At placement, there were no 
differences $(P>0.05)$ in chick weight between the three groups for either experimental replicate (no treatment control $\quad[\mathrm{NTC}]=0.044 \pm 0.001 \mathrm{~kg} ; \quad 300 \mathrm{~g} / \mathrm{MT}=0.045 \pm$ $0.0003 \mathrm{~kg} ; 500 \mathrm{~g} / \mathrm{MT}=0.044 \pm 0.001 \mathrm{~kg}$ ). Group weights were determined at the conclusion of each study, and supplement-fed chicks were slightly heavier $(300 \mathrm{~g} / \mathrm{MT}=$ $0.525 \pm 0.007 \mathrm{~kg} ; 500 \mathrm{~g} / \mathrm{MT}=0.526 \pm 0.002 \mathrm{~kg}$ ) than the NTC chicks $(0.522 \pm 0.003 \mathrm{~kg})$; however, these differences were not significant $(P>0.05)$.

\section{Alpha diversity analysis}

For each independent experiment $(n=2)$, five ileal and jejunal samples were collected. In total, samples from 10 chickens were included in the bioinformatics analyses per treatment for all analytics. Evenness and richness are two essential components to alpha diversity. Therefore, taken together, both metrics are able to assess changes in alpha diversity due to location or treatment. The effects of location were significant $(P<0.05)$ for Shannon's diversity index (Fig. 1a) and Pielou's evenness (Fig. 1b) comparing the ileum and jejunum. There was a significant $(P<0.05)$ decrease in Shannon's diversity index and Pielou's evenness metric for the ileum. Meaning, species richness and the even distribution of that richness across the ileum is less than that of the jejunum.

\section{Beta diversity analysis}

For each study $(n=2)$, a total of 5 samples were collected. In total, samples from 10 chickens were included in the bioinformatics analyses per treatment for all analytics. Specific to beta-diversity, the qualitative metrics Bray-Curtis Dissimilarity Index (Fig. 2a) and Weighted Unifrac Distance Matrix (Fig. 2b) were statistically $(P<$ $0.05)$ significant for the interaction of treatment and location. The statistical outputs for Bray-Curtis and weighted unifrac distance matrix are shown in Tables 1 and 2 , respectively. There is a clear difference in beta diversity for both matrices between the ileum and jejunum (Table 1; $\mathrm{Q}<0.05$ ). Additionally, there are significant changes to diversity between the tissue within treatment (Table 2; $\mathrm{Q}<0.05$ ). Specific to the comparison between the 300 and $500 \mathrm{~g} / \mathrm{MT}$ treatments, the $500 \mathrm{~g} / \mathrm{MT}$ treatment was statistically significant between the ileum and jejunum $(\mathrm{Q}=0.024)$. The $300 \mathrm{~g} / \mathrm{MT}$ treatment also exhibited this difference. Likely, the effects of the local microbiota drive these differences, as indicated by the alpha diversity analysis.

\section{Analysis of communities of the microbiota (ANCOM)}

For each study $(n=2)$, a total of 5 samples were collected. In total, samples from 10 chickens were included in the bioinformatics analyses per treatment for all analytics. Because of the qualitative differences in beta diversity, and how there could be tissue-specific effects driving these differences, it became necessary to sort the data using ANCOM (Analysis of Communities of the Microbiota) to delineate the potential changes to compositional diversity. In the ileum, there was no difference in treatment by organ. Therefore, the differences in treatment observed in the beta diversity index are likely due to tissue-specific effects, not the localized effect of treatments. However, there were dose-dependent responses observed in the jejunum at the family level (Fig. 3). Lactobacilliaceae predominated for all three treatment groups, with a stepwise increase in this population from the NTC to the $300 \mathrm{~g} / \mathrm{MT}$ and finally to the 500 g/MT group (Fig. 3a, b, c, respectively). This corresponds with a stepwise decrease in Staphylococcaceae from $3 \%$ in the NTC, $2 \%$ in the $300 \mathrm{~g} / \mathrm{MT}$ and $0 \%$ (rounded number) of the total operational taxonomic units (OTU) associated with treatment at $500 \mathrm{~g} / \mathrm{MT}$. In relation to NTC (35\%), the Enterobacteriaceae populations decreased in the $500 \mathrm{~g} / \mathrm{MT}(31 \%)$ while the $300 \mathrm{~g} /$ MT increased (37\%). In both the $300 \mathrm{~g} / \mathrm{MT}$ and $500 \mathrm{~g} /$ MT treatments, the OTU identified as Aerococcaceae did not fluctuate $(0 \%)$ compared to $7 \%$ in the NTC treatment. In relation to the three dietary treatments, Ruminococcaceae were more abundant in the $300 \mathrm{~g} / \mathrm{MT}(2 \%)$ treatment compared to the samples collected from the NTC $(0 \%)$ and $500 \mathrm{~g} / \mathrm{MT}(1 \%)$ treatments. Therefore, there were significant $(Q<0.05)$ changes in the microbial consortia statistically associated with the treatment groups in the jejunum. These effects did not occur in the ileum, which suggests that the substantial difference in microbial consortia between tissues likely drives the beta diversity effects observed for the ileum.

\section{Linear discriminant analysis effect size (LEfSE) analysis}

For each study $(n=2)$, a total of 5 samples were collected. In total, samples from 10 chickens were included in the bioinformatics analyses per treatment for all analytics. Least discriminant analysis effect size (LEfSE) accounts for underlying grouping by population; therefore, LEfSE indicates changes directly corresponding to treatment effects. As the $300 \mathrm{~g} / \mathrm{MT}$ treatment was the intermediary treatment, the NTC and $500 \mathrm{~g} / \mathrm{MT}$ scores were compared back to $300 \mathrm{~g} / \mathrm{MT}$ (Fig. 4). The linear discriminant analysis (LDA) score relative to a certain treatment has an inverse relationship with relative abundance. The NTC exhibited an increase in the LDA of Aerococcaceae, Gammaproteobacteria and Enterobacteriaceae populations relative to the $300 \mathrm{~g} / \mathrm{MT}$ group, which corresponds to a decrease in relative abundance (Fig. 4). Meanwhile, relative to $300 \mathrm{~g} / \mathrm{MT}$, the $500 \mathrm{~g} / \mathrm{MT}$ treatment group had a lower LDA in Clostridiaceae and Microccoaceae which translates to an increase in relative abundance (Fig. 4). When parsing out important veterinary pathogens, Enterobacteriaceae demonstrated a stepwise decrease in relative abundance 


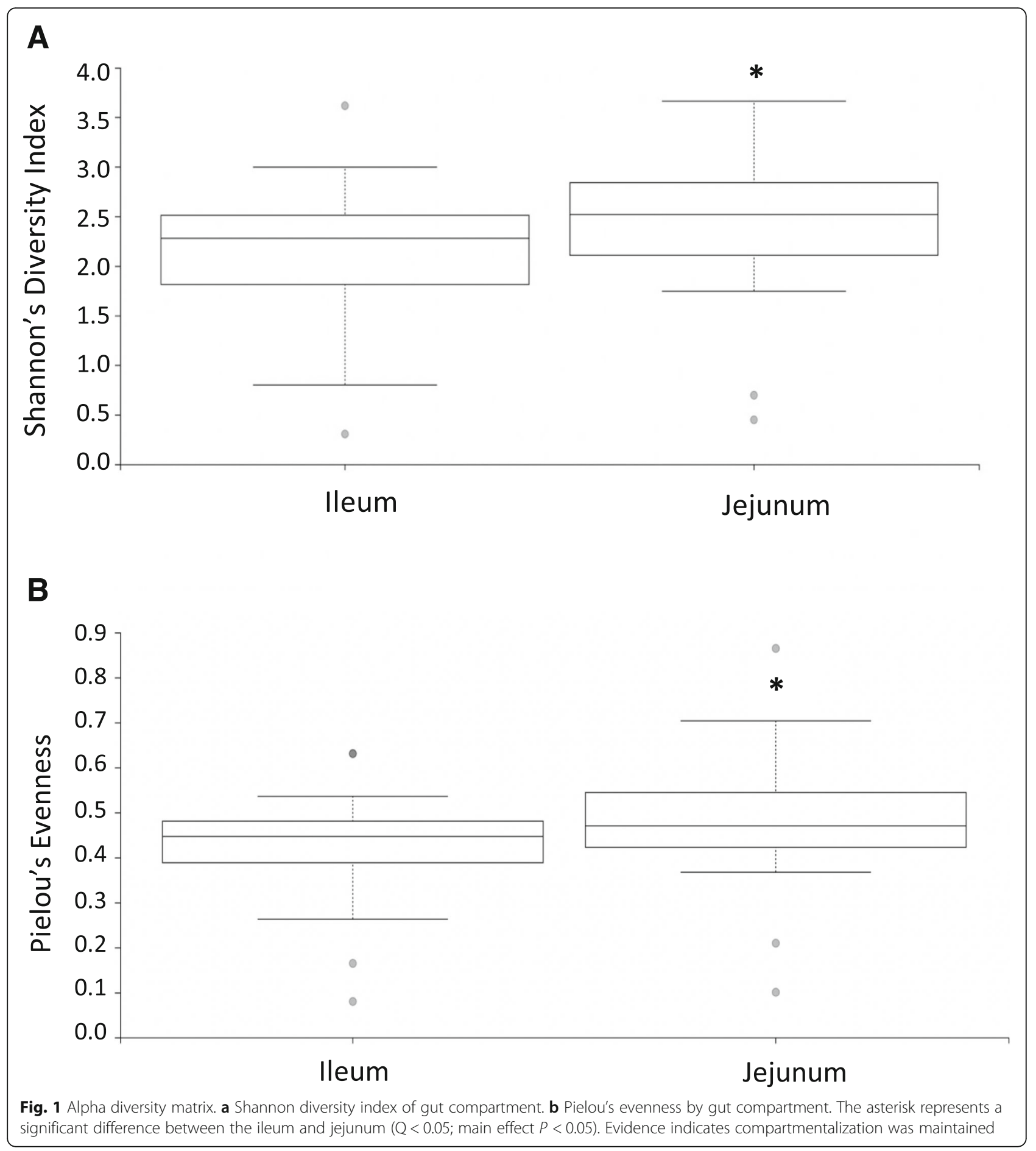

(from NTC onward) (Fig. 5). However, for Clostridiaceae, there was a significant increase in that relative population for $500 \mathrm{~g} / \mathrm{MT}$ compared to the NTC and $300 \mathrm{~g} / \mathrm{MT}$ treatments (Fig. 6), which is also supported by Fig. 4.

\section{Discussion}

There are numerous studies by our laboratory and others highlighting the benefit of using encapsulated ingredients for targeted release in the poultry GIT [10, $21,27,30-35]$. In a previous study, the blend of organic acids and botanicals evaluated herein enhanced gut immune and barrier function in the ileum and jejunum of weaned pigs [33]. In a separate study designed to begin to understand the mode-of-action at the gut level, a kinome analysis of ileal and jejunal samples collected from chickens revealed both common and distinct 
A

Axis $2(25.12 \%)$

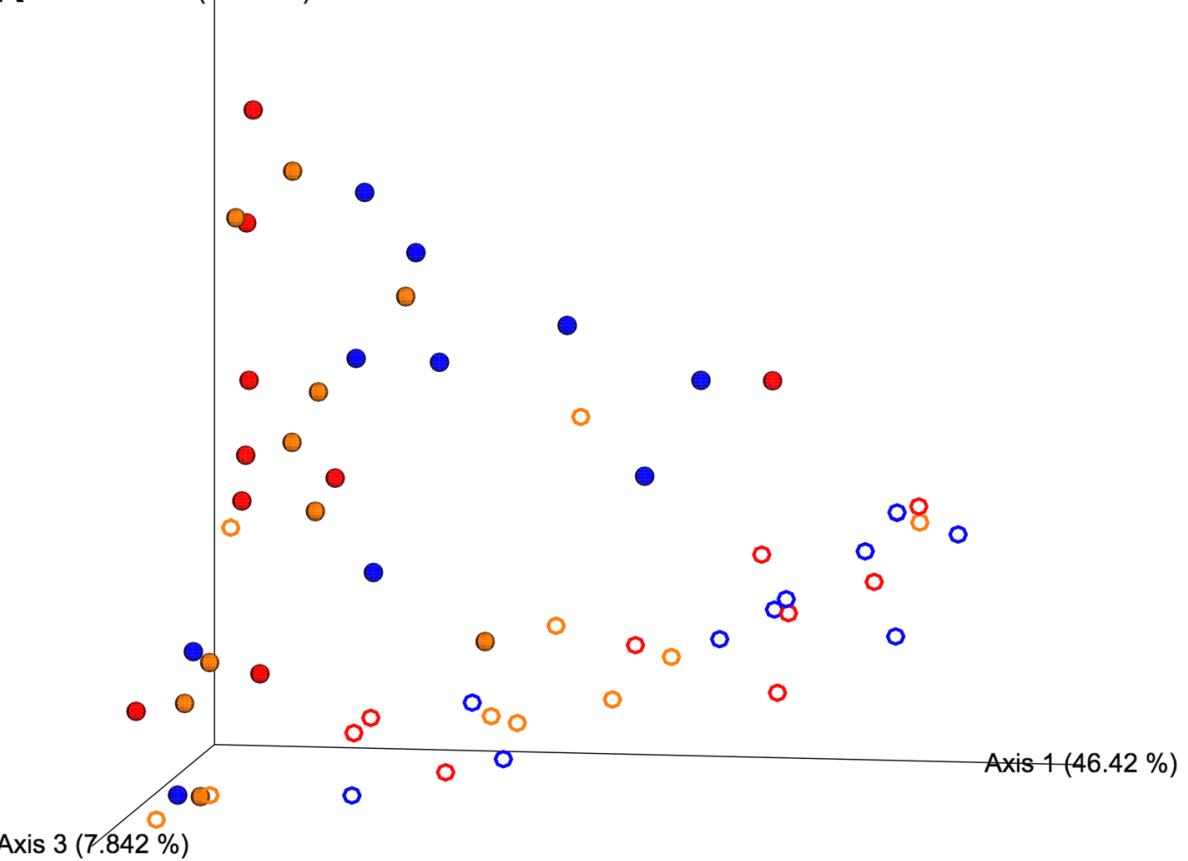

B

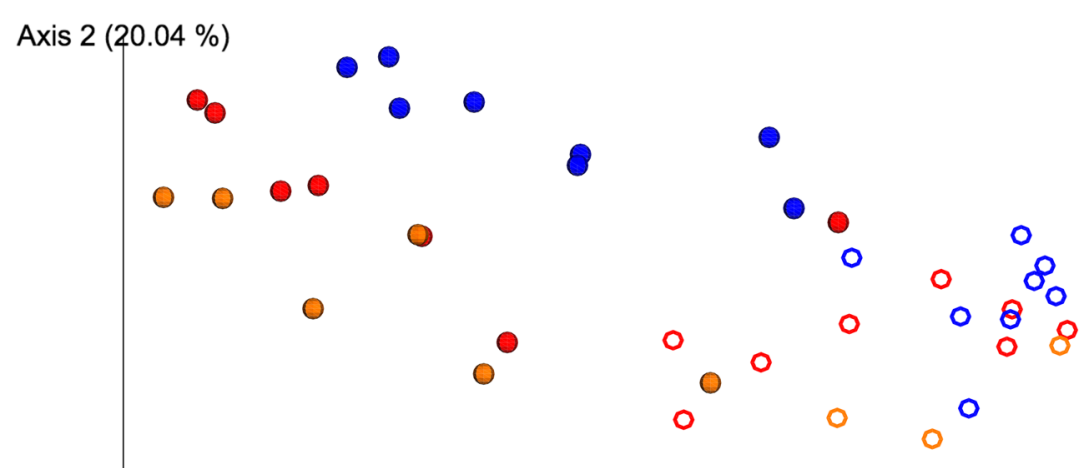

Axis $3(10.57 \%)$

Fig. 2 Beta diversity matrix. a Weighted unifrac distance matrix. b Bray-Curtis dissimilarity index. Shape coding: Sphear: Ileum; Ring: jejunum. Color: Red: NTC; Blue: $300 \mathrm{~g} / \mathrm{MT}$; Gold: $500 \mathrm{~g} / \mathrm{MT}$. Significant differences exist for compartmentalization. The effect of treatment was demonstrated throughout the study 
Table 1 Bray-Curtis dissimilarity index statistical output

\begin{tabular}{|c|c|c|c|c|c|c|}
\hline Group 1 & Group 2 & Sample Size & Permu-tations & $R$ value & $p$-value & q-value* \\
\hline 0 lleum & 0 Jejunum & 10 & 999 & 0.64368999 & 0.001 & 0.00166667 \\
\hline 0 lleum & 300 lleum & 10 & 999 & 0.07736626 & 0.117 & 0.135 \\
\hline 0 lleum & 300 Jejunum & 10 & 999 & 0.73744856 & 0.001 & 0.00166667 \\
\hline 0 lleum & 500 lleum & 10 & 999 & 0.01646091 & 0.356 & 0.38142857 \\
\hline 0 lleum & 500 Jejunum & 10 & 999 & 0.61783265 & 0.001 & 0.00166667 \\
\hline 0 Jejunum & 300 Ileum & 10 & 999 & 0.38518519 & 0.001 & 0.00166667 \\
\hline 0 Jejunum & 300 Jejunum & 10 & 999 & -0.0292181 & 0.622 & 0.622 \\
\hline 0 Jejunum & 500 Ileum & 10 & 999 & 0.67530864 & 0.001 & 0.00166667 \\
\hline 0 Jejunum & 500 Jejunum & 10 & 999 & 0.19862826 & 0.014 & 0.01909091 \\
\hline 300 Ileum & 300 Jejunum & 10 & 999 & 0.45311111 & 0.001 & 0.00166667 \\
\hline 300 Ileum & 500 lleum & 10 & 999 & 0.13933333 & 0.024 & 0.03 \\
\hline 300 Ileum & 500 Jejunum & 10 & 999 & 0.50977778 & 0.001 & 0.00166667 \\
\hline 300 Jejunum & 500 Ileum & 10 & 999 & 0.74811111 & 0.001 & 0.00166667 \\
\hline 300 Jejunum & 500 Jejunum & 10 & 999 & 0.24144444 & 0.006 & 0.009 \\
\hline 500 Ileum & 500 Jejunum & 10 & 999 & 0.41222222 & 0.001 & 0.00166667 \\
\hline
\end{tabular}

*Bold indicates $\mathrm{q}<0.05$

signaling pathways and proteins that were activated in each tissue segment compared to control-fed chickens [30]. Specifically, the Rap1 signaling pathway was activated compared to ileal samples [30] which could contribute to intestinal homeostasis and enhanced barrier function [36]. However, neither of the above-mentioned studies considered the role and impact on the gut microbial ecology; therefore, in the present study we determined the microbial populations of the ileum and jejunum from supplement-fed chickens compared to controls to provide additional insight.
The microencapsulated blend of organic acids and botanicals used herein is recognized by the European Union Commission and European Food Safety Authority ([EFSA]; AviPlus ${ }^{\circ} \mathrm{P}$ EFSA identification number $4 \mathrm{~d} 3$ ) for its ability to enhance growth and feed efficiency in healthy chickens. In the current study, there were no weight differences $(P>0.05)$ between the supplementand control-fed chicks, but the chicks on the supplemented diets were slightly heavier than the NTC in a dose-dependent manner. The fact that there were no statistical differences in weight is not surprising since

Table 2 Weighted unifrac distance matrix statistical output

\begin{tabular}{lllllll}
\hline Group 1 & Group 2 & Sample Size & Permu-tations & R value & $p$-value & q-value* \\
\hline 0 lleum & 0 Jejunum & $\mathbf{1 0}$ & $\mathbf{9 9 9}$ & $\mathbf{0 . 4 4 7 8 7 3 8}$ & $\mathbf{0 . 0 0 2}$ & $\mathbf{0 . 0 0 6}$ \\
0 lleum & 300 lleum & 10 & 999 & 0.0436214 & 0.212 & 0.24692308 \\
0 lleum & 300 Jejunum & $\mathbf{1 0}$ & $\mathbf{9 9 9}$ & $\mathbf{0 . 5 8 5 7 3 3 8 8}$ & $\mathbf{0 . 0 0 2}$ & $\mathbf{0 . 0 0 6}$ \\
0 lleum & 500 lleum & 10 & 999 & -0.0633745 & 0.875 & 0.875 \\
0 lleum & 500 Jejunum & 10 & 999 & 0.24801097 & 0.017 & 0.02833333 \\
0 Jejunum & 300 lleum & $\mathbf{1 0}$ & $\mathbf{9 9 9}$ & $\mathbf{0 . 3 2 7 2 9 7 6 7}$ & $\mathbf{0 . 0 0 3}$ & $\mathbf{0 . 0 0 7 5}$ \\
0 Jejunum & 300 Jejunum & 10 & 999 & -0.0403292 & 0.679 & 0.7275 \\
0 Jejunum & 500 lleum & $\mathbf{1 0}$ & $\mathbf{9 9 9}$ & $\mathbf{0 . 4 5 4 5 9 5 3 4}$ & $\mathbf{0 . 0 0 1}$ & $\mathbf{0 . 0 0 5}$ \\
0 Jejunum & 500 Jejunum & 10 & 999 & 0.04389575 & 0.205 & 0.24692308 \\
300 lleum & 300 Jejunum & $\mathbf{1 0}$ & $\mathbf{9 9 9}$ & $\mathbf{0 . 4 5 0 2 2 2 2 2}$ & $\mathbf{0 . 0 0 1}$ & $\mathbf{0 . 0 0 5}$ \\
300 lleum & 500 lleum & 10 & 999 & 0.04577778 & 0.214 & 0.24692308 \\
300 lleum & 500 Jejunum & $\mathbf{1 0}$ & $\mathbf{9 9 9}$ & $\mathbf{0 . 2 3 6 2 2 2 2 2}$ & $\mathbf{0 . 0 0 8}$ & $\mathbf{0 . 0 1 7 1 4 2 8 6}$ \\
300 Jejunum & 500 lleum & $\mathbf{1 0}$ & $\mathbf{9 9 9}$ & $\mathbf{0 . 6 2 4}$ & $\mathbf{0 . 0 0 1}$ & $\mathbf{0 . 0 0 5}$ \\
300 Jejunum & 500 Jejunum & 10 & 999 & 0.11466667 & 0.075 & 0.1125 \\
500 lleum & 500 Jejunum & $\mathbf{1 0}$ & $\mathbf{9 9 9}$ & $\mathbf{0 . 2 2 2 4 4 4 4 4}$ & $\mathbf{0 . 0 1 3}$ & $\mathbf{0 . 0 2 4 3 7 5}$ \\
\hline
\end{tabular}

*Bold indicates $\mathrm{q}<0.05$ 


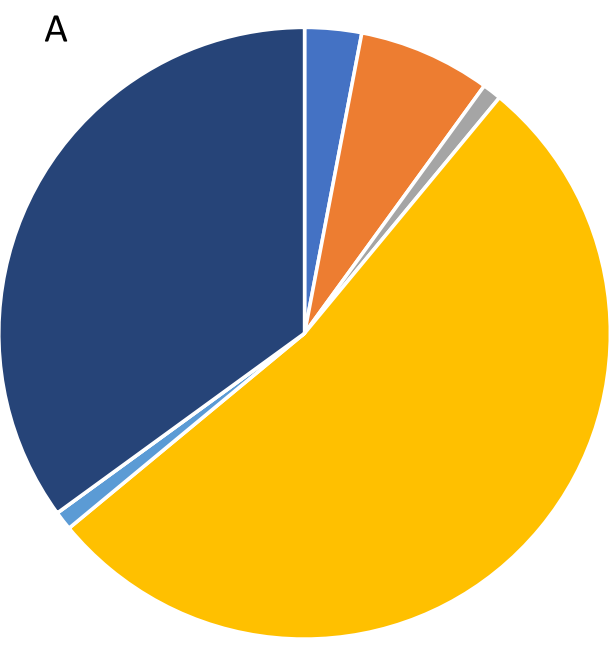

- Staphylococcaceae (3\%)

Aerococcaceae (7\%)

Enterococcaceae (1\%)

Lactobacillaceae (53\%)

neptostreptococcaceae (1\%)

\section{Ruminococcaceae (0\%)}

- Enterobacteriaceae (35\%)

B.

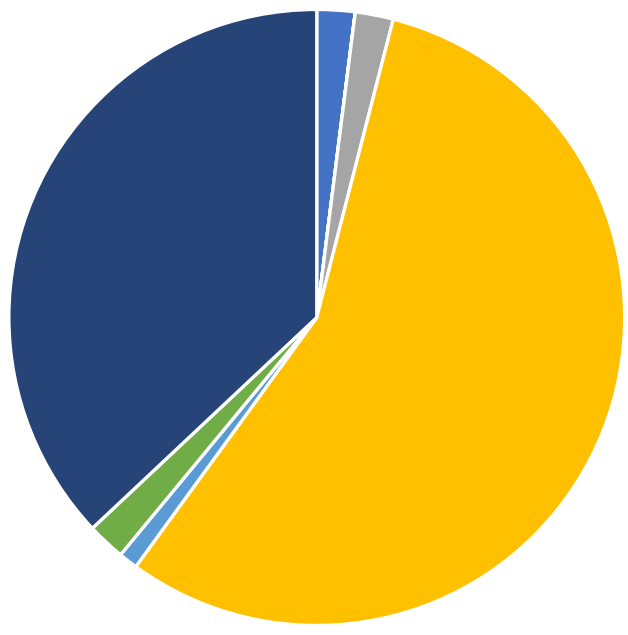

- Staphylococcaceae (2\%)

- Aerococcaceae (0\%)

- Enterococcaceae (2\%)

- Lactobacillaceae (56\%)

- Peptostreptococcaceae (1\%)

- Ruminococcaceae (2\%)

- Enterobacteriaceae (37\%)

C

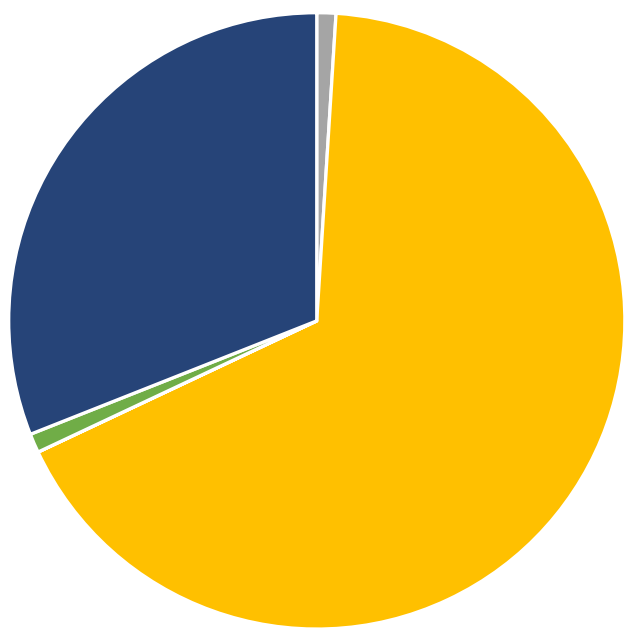

- Staphylococcaceae (0\%)

- Aerococcaceae (0\%)

- Enterococcaceae (1\%)

- Lactobacillaceae (67\%)

- Peptostreptococcaceae (0\%)

- Ruminococcaceae (1\%)

- Enterobacteriaceae (31\%)

Fig. 3 Analysis of communities of the microbiota (ANCOM) for jejunum samples. a NTC; b $300 \mathrm{~g} / \mathrm{MT}$; c $500 \mathrm{~g} / \mathrm{MT}$. The legends for the specific operational taxonomic units (OUT) associated with treatment as defined by ANCOM $(Q<0.05)$ is listed on the figure. Significant fluctuations occurred with increasing inclusion of the microencapsulated blend of organic acids and botanicals to the broiler diet 


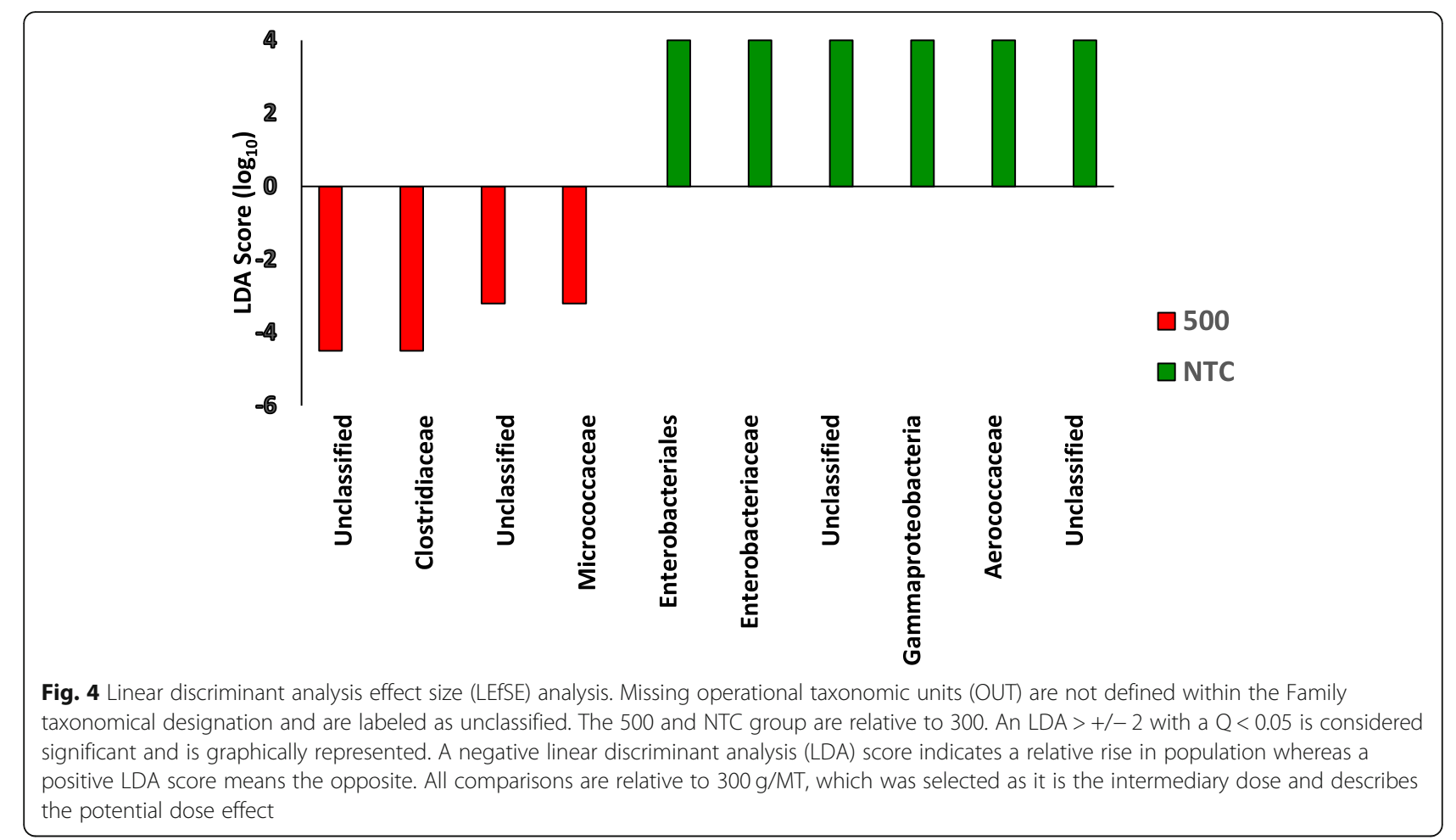

the study was terminated at 15 days which is similar to another feed additive study carried out for 14 days [37]. Broiler studies focused on growth and performance are typically carried out to 35-42 days with incremental measures incorporated into the study design [10, 28, 38]; however our findings do suggest early shifts in the jejunal microbial populations and signaling pathways [30] could be contributing to the enhanced growth and performance that was observed during the EFSA approval process. In fact, in a study conducted on broilers fed with incremental doses of the same microencapsulated blend of organic acids and botanicals demonstrated a linear increase of body weight and improvement of feed efficiency starting at 7 days and reaching the maximum

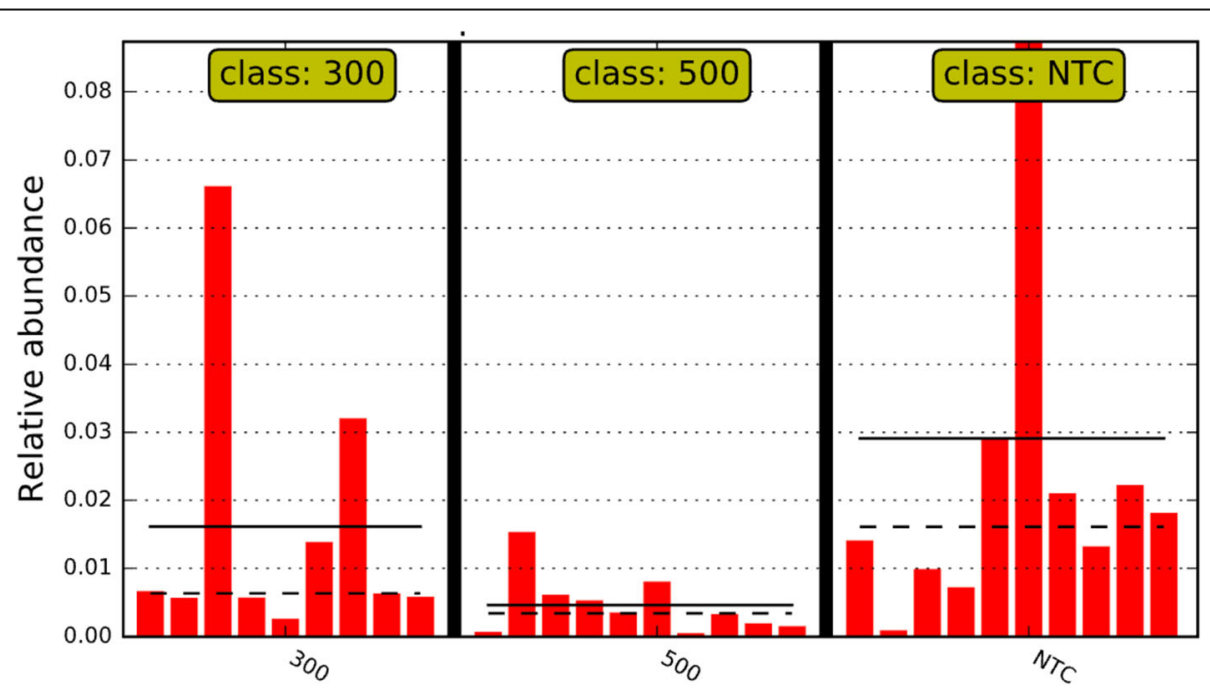

Fig. 5 Enterobacteriaceae linear discriminant analysis effect size (LEfSE) relative abundance. The dotted lines are the median and the solid lines are the class mean. The relative abundance significant by LEfSE of each animal is displayed. The NTC has on average a greater abundance of Enterobacteriaceae, with a stepwise decrease in this population with increasing inclusion of the microencapsulated blend of organic acids and botanicals to the broiler diet 


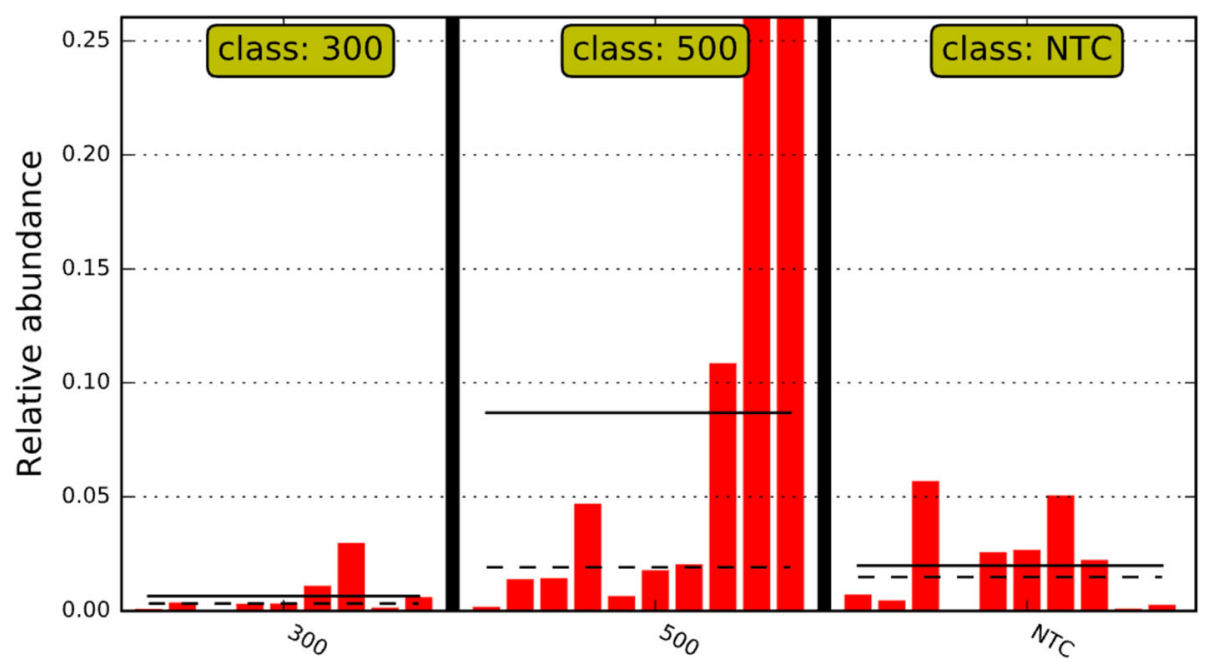

Fig. 6 Clostridiaceae linear discriminant analysis effect size (LEfSE) relative abundance. The dotted lines are the median and the solid lines are the class mean. The relative abundance significant by LEfSE of each animal is displayed. The $300 \mathrm{~g} / \mathrm{MT}$ treatment has on average a lower abundance of Clostridiaceae, with an increase at the $500 \mathrm{~g} / \mathrm{MT}$ level

effect at 35 days [29]. The performance results were correlated to an increase in Lactobacillus counts in the feces thus suggesting the change in microbial populations as one of the factors contributing to the overall result at the completion of the grow-out. Nevertheless, microbial populations are just one of the factors determining growth performance as there are also important contributions coming from the host, the environment, and the complex group of interactions among these elements. As one study cannot unveil and explain the complexity of all of these interactions, with the present study we wanted to understand the impact of microencapsulated organic acids and botanicals on the jejunum and ileum populations at the early stages of development of broiler chickens irrespective of growth performance at the end of grow-out. The limitation of this kind of approach resided in the lack of correlation between the changes we observed at day 15 days and the impact these might have had on the final performance. To more definitively address the correlation between microbial populations and performance, time course studies with additional biological replicates and increased numbers of chicks need to be conducted through the entire growout. Poultry feed additive studies typically focus on one or more aspect of performance (body weight or feed efficiency); however, the objective of the current project sought to follow-up on the earlier kinome study where signaling differences were observed at the tissue level (ileum versus jejunum) which, as we have shown herein, tissue-specific differences were also observed in the microbial populations.

Evidence suggests the stability of the microbiota is defined over time; however, changes observed in stable systems by compartment (tissue segment) can also indirectly support what is or is not viewed as a stable microbiota that may contribute to a loss in homeostasis $[39,40]$. A classic example of the breakdown of gastrointestinal homeostasis is the emergence of ecological dysbiosis resulting in the de-compartmentalization of the gastrointestinal microbiota $[19,41]$. The current study was not focused specifically on homeostasis or dysbiosis over time, but the findings herein indicate dietary supplementation with organic acids and natural compounds did result in significant compartmentalization of the microbial ecology within the ileum and jejunum of chickens. Each compartment functions independently with nutrient digestion and absorption typically occurring in the jejunum with water and mineral adsorption generally taking place in the ileum $[42,43]$; therefore, it would be expected that the microbial populations would, in fact, differ between the two compartments. Additionally, while tissue differences in the microbial makeup exist comparing the NTC to tissue from supplement-fed chickens, there is not a collapse and shrinkage in diversity or a bloom of populations. These data are in agreement with other studies looking into nutrition and gastrointestinal health studies $[39,40]$. While we cannot speak to potential changes or stability over time, data presented does indicate the microbiota is biologically diverse at $15 \mathrm{~d}$-of-age in chicks provided a diet supplemented with the microencapsulated blend of organic acids and botanicals. However, some authors suggest the gut and microbiota at $15 \mathrm{~d}$-of-age is only semi-developed [44]; therefore, future studies should consider the microbiota populations over the typical 42-day grow-out period.

Alpha diversity speaks to the community structure and evenness of the microbial ecosystem without taking into 
account differences in speciation while Shannon's diversity index is classically associated with numerous microbial studies and is used to calculate evenness [16]. Beta diversity indicates there may be compositional differences that are arising, with Bray-Curtis being a function of total assessment and the Weighted Unifrac Distance Matrix considering phylogenetic branch length and both are considered qualitative as total reads and counts leading to the differences are not considered [16]. Dietary supplementation with natural compounds including organic acids and essential oils does not always result in changes to alpha and beta diversity in microbial populations within the poultry GIT [10]. However, the blend of organic acids and botanicals used in the current study, produced an increase in diversity and evenness for the jejunum compared to the ileum. Similarly, in other pharmacological studies, the biotransformation of drugs by the microbiota results in their absorption in the jejunum and are linked to increased diversity and biological activity of the microbial population $[45,46]$. The jejunum is the main sight for nutrient absorption in poultry [42], as well as in mammals, and it has been suggested that the jejunum is the most logical site to observe treatment effects [13] which is what we observed in the current study. Also, some feed additive studies utilize traditional culture-dependent microbiological evaluation to characterize the GIT microbial populations $[28,38,47]$. While these studies are valid and valuable, they are unable to take into account compositional and diversity changes. Therefore, the culture-independent study herein provides a deeper insight into the complete microbial shifts in two diverse and bioactive components of the GIT.

Natural compounds such as oregano and its derivatives, including thymol and carvacrol, are recognized for their potential benefits to the poultry industry because of antimicrobial properties and animal health benefits [9]. Additionally, dietary supplementation with thymol has shown to increase Lactobacillus populations in the ileum [24, 47]; however, in the current study the changes in Lactobacillus populations were more pronounced in the jejunum compared to the ileum. This dissimilarity is likely attributed to experimental design differences including, but not limited to, the delivery method (non-encapsulated vs encapsulated), the genetic line of chickens used (Arbor Acre vs Cobb), the feed additive, or the thymol concentration (25\% vs $1.7 \%$ ). Even though the tissue-specific changes were different than the aforementioned study, our findings are in agreement with another study showing that inclusion of thymol does alter the GIT microflora of poultry [23]. Another natural compound, a green tea component, also resulted in increased Lactobacillus in the jejunum compared to the ileum when fed to chickens [48]. Collectively, these studies indicate an important role for the inclusion of thymol and other natural compounds into the diet as antibiotic alternatives.

In addition to increased Lactobacillus populations, other favorable changes were observed following supplementation including significant changes in Clostridiaceae in the $500 \mathrm{~g} / \mathrm{MT}$ jejunal samples. Similarly, supplementation with eugenol, an essential oil, increased members of the Clostridiales order in mice that proved protective against pathogenic challenge [49]. There are a number of studies employing supplementation with natural products including organic acids and phytochemicals that show improvements to intestinal integrity as well as protecting against the pathology and loss of performance associated with necrotic enteritis in broilers $[10,24,35$, 50]. Future challenge trials will be conducted to determine if the blend of organic acids and botanicals used herein confers protective effects against Clostridium perfringens-induced necrotic enteritis and what role the GIT microbial populations play in determining disease outcome. Ruminococcaceae families (300 and $500 \mathrm{~g} / \mathrm{MT}$ ) also increased in our study that was accompanied by a decrease in Enterobacteriaceae (in the $500 \mathrm{~g} / \mathrm{MT}$ dose). These data are in agreement with recent studies that also fed diets that incorporated an encapsulated blend of organic acids and essential oils [10] and phytonutrients [49]. The organic acids and essential oils were different, but the beneficial effects were similar which is also supported by numerous studies using diverse organic acids including, but not limited to, butyric acid [38], encapsulated benzoic acid [27], or formic and propionic acids [28] to enhance the GIT microbiota, poultry health, and performance. Collectively, the data presented herein, along with supporting studies in the literature, demonstrate the importance of targeted release of natural compounds in the poultry GIT to maximize efficacy and potential benefits to the bird. It has been said "increased understanding of how the microbiota interacts with animal hosts will improve microbiome intervention strategies to mitigate production losses" [51]. This statement becomes even more critical as antibiotic use is further curtailed and restricted within the poultry industry, and the present study begins to understand the hostmicrobiome interaction in the presence of natural antibiotic alternatives.

As with any laboratory-controlled experiment, there are limitations that prohibit the inclusion of all variables encountered on the farm under commercial conditions. One of the most obvious discrepancies would be the environment where the newly hatched chick is placed. Under commercial conditions chicks would be placed in a house that has been exposed to thousands of chickens compared to an experimental room that is thoroughly disinfected prior to placement of chicks onto clean litter 
versus some commercial settings where chicks would be placed on used litter. Clearly, these differences would likely impact the outcome of a microbiome experiment due to the immediate exposure to the myriad of microorganisms found in a poultry house; however, this does not diminish our findings as clear compartmentalization of the microbial populations between the ileum and jejunum were observed. One approach to mitigate this experimental limitation would be to place the chicks on used litter to more realistically mimic the early GIT colonization seen under commercial conditions; however, this approach introduces uncontrollable variables making reproducibility of results difficult. With respect to the microbiome analysis approach that was employed, one of the limitations is the results are qualitative which does not take into consideration cell counts and $16 \mathrm{~S}$ ribosomal DNA (rDNA) copy number. Different populations can contribute varying copy numbers of $16 \mathrm{~S}$ rDNA to the analysis; therefore, using quantitative methods will become more important and commonplace as microbiome studies evolve and technologies advance [52]. Additionally, the ability to utilize long read technology will also become necessary to truly understand microbial shifts due to treatment, instead of sequencing small variable regions, such as V3 or V4; however, the approaches we employed are widely accepted and are common practice today $[48,53]$. Despite the above-mentioned limitations, the observed changes in beta diversity will remain consistent and are indicative of potentially optimal microbiota changes. Further, this study demonstrated that shifts in dispersion and mean, as analyzed by ANISOM, occurred by treatment. This type of metric will also stand the test of time and prove essential in delineating the biological role of the microbiota and how it is affected by treatment. It should also be noted that these limitations exist with all currently conducted microbiota studies that are not commercially derived, with many of these limitations existing for decades. Yet, studies such as the one conducted here are considered academically sound and important for the industry. While microbiota studies will become more advanced as technology and bioinformatics improves, the importance of academically derived studies independent of field conditions will always be important and relevant.

Future studies considering the impact of the biochemical and/or metabolites produced in each compartment of the GIT would provide additional mechanistic insight. Studies in the literature show changes to the microbial populations could diffuse outward or that the metabolites are further transformed by downstream microbial populations impacting colonization by foodborne pathogens such as Salmonella [54, 55]. Dietary supplementation with organic acids and botanicals significantly lowers Salmonella [31] and Campylobacter [32] colonization in market-age broilers. Though not considered in those earlier studies, it is possible that changes to the GIT microbial populations while the bird is developing could have contributed to the observed decreases in Salmonella and Campylobacter colonization, but additional studies are required to confirm this hypothesis. Studies support there is compartmental activation of the microbiota; but ultimately it will be the resulting physiological effects within the different compartments as they carry out their specific biological processes $[46,56]$ that will have the greatest impact.

Finally, although outside the scope of the current manuscript, the authors recognize the importance of determining feed efficiency, nutrient absorption, and other GIT functionality traits as predictors and contributors to the final growth performance and on-farm profitability.

\section{Conclusions}

The bioactive site for the microencapsulated blend of organic acids and botanicals used in this study is in the jejunum, which is also the site of nutrient absorption. Understanding these fundamental changes to the microbiota composition of the ileum and jejunum indicate future studies should consider evaluating the metabolome which will provide a deeper understanding of the impact of organic acids and botanicals. However, based on the changes shown herein, the data indicate inclusion of the microencapsulated blend of organic acids and botanicals does enhance the GIT microbiota and may be a viable antibiotic alternative for use in the poultry industry.

\section{Methods}

\section{Experimental design, animal husbandry, and tissue collection}

The experiments were conducted in accordance with the recommended code of practice for the care and handling of poultry and followed the ethical principles according to the Guide for the Care and Use of Laboratory Animals [57]. All bird studies were under the approved experimental procedures outlined in protocol \#2017008 and were approved by the USDA/ARS Institutional Animal Care and Use Committee and overseen by Dr. Roger B. Harvey, DVM (attending veterinarian).

Day-of-hatch by-product male breeder chicks were obtained from a commercial hatchery (Timpson, TX, USA), and were not vaccinated at any point during the study. The chicks were transported in standard chick boxes and placed in a BL2 building in floor pens $(3 \mathrm{~m} \times$ $3 \mathrm{~m})$ containing wood shavings and provided supplemental heat and ad libitum access to food supplied in hanging feeders and fresh water through nipple drinkers. Chickens were provided $24 \mathrm{~h}$ of continual light at placement to ensure sufficient water and food intake, then transitioned to $18 \mathrm{~h}$ of light and $6 \mathrm{~h}$ of darkness for the remainder of the study. The temperature of the pens 
was maintained at $35^{\circ} \mathrm{C}$ for day 1 to 3,32 to $34^{\circ} \mathrm{C}$ for day 4 to 7 , and 29 to $31^{\circ} \mathrm{C}$ for day 8 to 15 . Chickens were monitored each morning (08:00) for mortality, behavioral changes, litter quality, and feed and waterers were checked to ensure they were in proper working order. No mortality, behavioral changes, or other animal welfare concerns were observed during the study. The chicks were not treated with any medications or other therapeutic interventions during the study. No antibiotics were given to the chicks nor included in any of the diets used in the study.

Two independent trials were conducted using chicks from a different hatch-out. The chicks were weighed at placement (day of hatch) and at the conclusion of the study (d15). Chickens from the two hatches were maintained separately to ensure proper biological replication of the experiment. The two replicates of the experiment were handled as follows: chickens $(n=15)$ were randomly selected and placed into one of three groups: the NTC $\left(0 \mathrm{~g} / \mathrm{MT}\right.$ AviPlus ${ }^{\circ} \mathrm{P} ; n=5$ chickens $)$ or one of the experimental groups $(300 \mathrm{~g} / \mathrm{MT} ; n=5$ chickens; $500 \mathrm{~g} / \mathrm{MT}$ AviPlus ${ }^{\circ} \mathrm{P} ; n=5$ chickens). The experiment was conducted using two replicate pens therefore 10 chickens/treatment were used for all analyses. Chickens assigned to the control pen were allowed ad libitum access to a balanced, unmedicated, antibiotic-free corn and soybean mealbased starter diet that met or exceeded the established nutrient requirements [58]. Chickens assigned to the supplement-fed pens were given free access to the same starter diet mixed with 300 or $500 \mathrm{~g} /$ metric ton (MT) of a microencapsulated blend of citric (25\%) and sorbic (16.7\%) acids, thymol (1.7\%), and vanillin (1.0\%) (AviPlus ${ }^{\circ}$, Vetagro S.p.A., Reggio Emilia, Italy). The remaining $55.6 \%$ of the feed additive is comprised of hydrogenated vegetable fats. The feed was mixed in small batches for $15 \mathrm{~min}$ (34 g AviPlus ${ }^{\circ} \mathrm{P} / 113 \mathrm{~kg}$ feed and $56.7 \mathrm{~g}$ AviPlus ${ }^{\circ} \mathrm{P} / 113 \mathrm{~kg}$ feed for the 300 and $500 \mathrm{~g} / \mathrm{MT}$, respectively) using a Wenger $\mathrm{AB}$ batch mixer (Sebetha, KS). The control diet was mixed first to ensure consistency of the mash supplied to each group of chicks.

All chickens assigned to the control pens were evaluated first followed by those in the 300 and $500 \mathrm{~g} / \mathrm{MT}$ groups. For both experimental replicates, chickens on the control, 300 , and $500 \mathrm{~g} / \mathrm{MT}$ diet $(n=5$ per group/experiment; $n=10$ total) were euthanized by cervical dislocation and necropsied at 15-days-of-age. The ileum and jejunum were selected because they are two important organ systems associated with feed efficiency and production in broilers. In relation to Meckel's diverticulum, the jejunum sample was collected approximately $10 \mathrm{~cm}$ proximal and the ileum sample was collected approximately $10 \mathrm{~cm}$ distally. Total content from these regions of the jejunum and ileum were collected and immediately flash frozen in liquid nitrogen to preserve activity followed by transfer to $-80^{\circ} \mathrm{C}$ until further processing and analysis. Samples were collected at day 15 based on previous work [33, 34] and in consideration of the productive cycle of broilers. In commercial settings, most diet changes going from the starter to grower occurs between 10 and 15 daysof-age. The first two weeks are very critical to the development of the gastrointestinal and immunological function and by 2 to 3 -wk-of-age broilers have a diversified microflora.

\section{DNA extraction}

The DNA was extracted and sequenced as per standard laboratory guidelines [59]. Briefly, the tissue (ileum or jejunum) contents were thawed, homogenized, and $0.3 \mathrm{~g}$ removed followed by extraction using the Qiagen Stool Kit (Qiagen, Hilden, Germany). The DNA was eluted and stored at $-20^{\circ} \mathrm{C}$ until the library preparations commenced. Using the amplicon sequence variance index primers and protocol, the library was prepared as previously described [60]. Normalization and library clean-up were also performed prior to sequencing $[59,60]$. The Illumina MiSeq $16 \mathrm{~S}$ rDNA Microbiome Library (version 2; Illumina, San Diego, CA, USA) was constructed and sequenced as per standard company guidelines. The sequences were exported from Illumina BaseSpace [61], demultiplexed, and prepared for import into QIIME2.2019.1 (quantitative insights into microbial ecology) [62].

\section{Microbiome analyses}

Each bird sample was handled on an individual basis $(n=10)$ and each tissue (ileum and jejunum) was kept separate for all analyses. The sequences were filtered for quality and chimera using divisive amplicon denoising algorithm (DADA2), with Q30 being the cut off range for sequence quality [63]. Additionally, in order to remove any potential chimeras that escaped detection, OTUs with a frequency of less than 3 were removed from the analyses. Alpha and beta analyses were performed using the standard QIIME2.2019.1 pipeline, with ANOSIM (analysis of similarities) selected as it considers dispersion and the mean difference in beta diversity per group. To refine the analyses, the tissue data was then sorted into either a "ileum" or "jejunum" dataset for compositional analysis. Differential abundance was evaluated using the plugin ANCOM [64], which considers the compositional changes associated with treatment. Finally, LEfSE analysis was performed per standard practices [65] to determine which populations were enriched by treatment using LDA, which is inversely related to ANCOM data [66]. 


\section{Statistical analyses}

Compositional microbiota studies are necessarily heterogeneous and represent the changes of a microbial consortia and structure by treatment. Therefore, the use of statistically sound plugins to evaluate the compositional data are important as standard statistical practices are irrelevant if they do not take into account the compositional nature of the data. Alpha and beta diversity parameters were considered significant if the main effect was $P<0.05$. Pairwise differences between the main effect of treatment were considered significant if $\mathrm{Q}<0.05$, which takes into account the false discovery rate associated with this class of data. The Kruskall-Wallis test was used in the alpha diversity metrics, meanwhile the ANISOM test was used for the beta diversity tests as per the standard QIIME2.2019.1 pipeline. The Q-value is representative of the corrected $p$ value, which is a standard component of multivariate and multihypothesis-based testing associated with this kind of data set. Finally, for ANCOM, PROC GLM was used in the background, with the central $\log 2$ ratio of the effect (W) significant of $Q<0.05$ evaluating the changes in the microbial consortia by treatment. Therefore, any OTUs arising from the analyses fluctuate statistically by treatment and are not quantitative differences between each treatment as the entirety of the microbial consortia fluctuation by treatment is what is regarded as significant in this analysis $(Q<0.05)$. Pairwise differences between the treatment groups was instead performed by LEfSE, which is an independent analysis but provides species differences, which was considered significant if the LDA $>+/-2$ and $Q<0.05$ at the family level. Initially, the analysis was relative to the $300 \mathrm{~g} / \mathrm{MT}$ group and important microbial families associated with poultry production were identified for further analyses. It should also be noted that a negative LDA score relative to a comparison indicates an increase in relative abundance, with a positive score meaning the opposite.

\section{Abbreviations \\ ANCOM: Analysis of communities of the microbiota; ANOSIM: Analysis of similarities; DADA2: Divisive amplicon denoising algorithm; EFSA: European Food Safety Authority; GIT: Gastrointestinal tract; LDA: Linear discriminant analysis; LEfSe: Linear discriminant analysis effect size; NTC: No treatment control; OTU: Operational taxonomic unit; QIIME: Quantitative insights into microbial ecology; rDNA: Ribosomal DNA}

\section{Acknowledgements}

The authors thank M. Reiley Street, Jr. (U.S. Department of Agriculture, Agricultural Research Senvice [USDAVARS], College Station, TX) for outstanding technical support and assistance with daily animal care. The USDA is an equal opportunity provider and employer. Mention of trade names or commercial products in this publication is solely for the purpose of providing specific information and does not imply recommendation or endorsement by the USDA.

\section{Authors' contributions}

CLS, AP, EG conceived the study. KMF performed the microbiome analytics. KMF and CLS wrote the manuscript and prepared the paper for publication. MHK, SCR, AP and EG edited the manuscript. All authors read and approved the final manuscript.

\section{Funding}

This research was supported, in part, by Vetagro ([CLS] Agreement number 58-3091-8-005; https://www.vetagro.com) and the USDA/ARS (3091-32000035-00D; https://www.ars.usda.gov/research/project/?accnNo=430283). There was no additional external funding received for this study. Vetagro provided support in the form of salaries for AP and EG, and supplied the AviPlus feed amendment, but did not have any additional role in data collection and analysis, decision to publish, or preparation of the manuscript. USDA/ARS provided support in the form of salary for CLS and KMF but did not have any additional role in the study design, data collection and analysis, decision to publish, or preparation of the manuscript. The specific roles of these authors are articulated in the 'author contributions' section. All other authors declare no conflict of interest.

\section{Availability of data and materials}

Data is freely available at www.github.com/RickeLab/BMCMicroSubmission26 Feb2020. Please contact SCR if you request additional information.

\section{Ethics approval and consent to participate}

All bird studies were under experimental protocol \#2017008 and were approved by the USDA/ARS Institutional Animal Care and Use Committee (IACUC) and overseen by Dr. Roger B. Harvey, attending veterinarian. The IACUC operates under the Animal and Plant Health Inspection Service (APHI S) establishment number 334299.

\section{Consent for publication}

Not applicable.

\section{Competing interests}

AP and EG are Vetagro employees but did not participate in the analysis of this study. All other authors declare no conflict of interest.

\section{Author details}

${ }^{1}$ U.S. Department of Agriculture, Agricultural Research Service, Southern Plains Agricultural Research Service, $2881 \mathrm{~F}$ and B Road, College Station, TX 77845, USA. ${ }^{2}$ Meat Science \& Animal Biologics Discovery Program, Department of Animal and Dairy Sciences, University of Wisconsin, Madison, WI, USA. ${ }^{3}$ DIMEVET, University of Bologna, Ozzano Emilia, Bologna, Italy. ${ }^{4}$ Vetagro S.p.A, Reggio Emilia, Italy. ${ }^{5}$ Vetagro Inc., Chicago, IL, USA.

Received: 9 March 2020 Accepted: 9 October 2020

Published online: 02 November 2020

\section{References}

1. Hashemi SR, Davoodi H. Phytogenics as new class of feed additive in poultry industry. J Anim Vet Adv. 2010;9(17):2295-304.

2. Wallace RJ, Oleszek W, Franz C, Hahn I, Baser KH, Mathe A, et al. Dietary plant bioactives for poultry health and productivity. Br Poult Sci. 2010;51(4): 461-87.

3. Madhupriya V, Shamsudeen P, Manohar GR, Senthikumar S, Soundarapandiyan $\mathrm{V}$, Moorthy M. Phyto feed additives in poultry nutrition a review. Inter J Sci Environ Tech. 2018;7(3):815-22.

4. Yang $Y$, lji PA, Choct M. Dietary modulation of gut microflora in broiler chickens: a review of the role of six kinds of alternatives to in-feed antibiotics. Worlds Poult Sci J. 2009:65(1):97-114.

5. Yang $X$, Liu Y, Yan F, Yang C, Yang X. Effects of encapsulated organic acids and essential oils on intestinal barrier, microbial count, and bacterial metabolites in broiler chickens. Poult Sci. 2019;98(7):2858-65.

6. Kabera JN, Semana E, Mussa AR, He X. Plant secondary metabolites: biosynthesis, classification, function, and pharmacological properties. J Pharm Pharmacol. 2014;2:377-92.

7. Dias DA, Urban S, Roessner U. A historical overview of natural products in drug discovery. Metabolites. 2012;2(2):303-36.

8. Abd El-Hack ME, Alagawany M, Abdel-Moneim AE, Mohammed NG, Khafaga AF, Bin-Jumah $M$, et al. Cinnamon (Cinnamomum zeylanicum) oil as a potential alternative to antibiotics in poultry. Antibiotics (Basel). 2020;9(5):210.

9. Alagawany M, El-Hack ME, Farag MR, Shaheen HM, Abdel-Latif MA, Noreldin $A E$, et al. The usefulness of oregano and its derivatives in poultry nutrition. Worlds Poult Sci J. 2018;74(3):463-73.

10. Abdelli N, Perez JF, Vilarrasa E, Cabeza Luna I, Melo-Duran D, D'Angelo M, et al. Targeted-release organic acids and essential oils improve performance 
and digestive function in broilers under a necrotic enteritis challenge. Animals (Basel). 2020;10(2):259.

11. Dittoe DK, Ricke SC, Kiess AS. Organic acids and potential for modifying the avian gastrointestinal tract and reducing pathogens and disease. Front Vet Sci. 2018:5:216.

12. Yadav S, Jha R. Strategies to modulate the intestinal microbiota and their effects on nutrient utilization, performance, and health of poultry. J Anim Sci Biotechnol. 2019;10:2

13. Clemente JC, Ursell LK, Parfrey LW, Knight R. The impact of the gut microbiota on human health: an integrative view. Cell. 2012;148(6):1258-70.

14. Tremaroli V, Backhed F. Functional interactions between the gut microbiota and host metabolism. Nature. 2012;489(7415):242-9.

15. Le Roy Cl, Woodward MJ, Ellis RJ, La Ragione RM, Claus SP. Antibiotic treatment triggers gut dysbiosis and modulates metabolism in a chicken model of gastro-intestinal infection. BMC Vet Res. 2019;15(1):37.

16. Feye KM, Baxter MFA, Tellez-Isaias G, Kogut MH, Ricke SC. Influential factors on the composition of the conventionally raised broiler gastrointestinal microbiomes. Poult Sci. 2020;99(2):653-9.

17. Choi MS, Kim JK, Kim DH, Yoo HH. Effects of gut microbiota on the bioavailability of bioactive compounds from ginkgo leaf extracts. Metabolites. 2019;9(7):132

18. Bicas JL, Fontanille P, Pastore GM, Larroche C. Characterization of monoterpene biotransformation in two pseudomonads. J Appl Microbiol. 2008;105(6):1991-2001.

19. Marin L, Miguelez EM, Villar CJ, Lombo F. Bioavailability of dietary polyphenols and gut microbiota metabolism: antimicrobial properties. Biomed Res Int. 2015;2015:905215.

20. Ricke SC. Impact of prebiotics on poultry production and food safety. Yale J Biol Med. 2018;91:151-9.

21. Piva A, Pizzamiglio V, Morlacchini M, Tedeschi M, Piva G. Lipid microencapsulation allows slow release of organic acids and natural identical flavors along the swine intestine. J Anim Sci. 2007;85(2):486-93.

22. Grilli E, Bodin JC, Gatta PP, Tedeschi M, Piva A, editors. Microencapsulation allows slow release of organic acids in the GI tract of broilers. Proc 16th Eur Symp Poult Nutr. Strasbourg: CABI Publishing; 2007.

23. Abd El-Hack ME, Alagawany M. Performance, egg quality, blood profile, immune function, and antioxidant enzyme activities in laying hens fed diets with thyme powder. J Anim Feed Sci. 2015;24:127-33.

24. Yin D, Du E, Yuan J, Gao J, Wang Y, Aggrey SE, et al. Supplemental thymol and carvacrol increases ileum Lactobacillus population and reduces effect of necrotic enteritis caused by Clostridium perfringes in chickens. Sci Rep. 2017;7(1):7334.

25. Govindasami T, Pandey AC, Palanivelu N, Pandey A. Synthesis, characterization and antibacterial activity of biologically important vanillin related hydrazone derivatives. Int J Organic Chem. 2011;01(03):71-7.

26. Harini ST, Kumar HV, Rangaswamy J, Naik N. Synthesis, antioxidant and antimicrobial activity of novel vanillin derived piperidin-4-one oxime esters: preponderant role of the phenyl ester substituents on the piperidin-4-one oxime core. Bioorg Med Chem Lett. 2012;22(24):7588-92.

27. Yousaf MS, Goodarzi Boroojeni F, Vahjen W, Manner K, Hafeez A, Ur-Rehman H, et al. Encapsulated benzoic acid supplementation in broiler diets influences gut bacterial composition and activity. Br Poult Sci. 2017;58(2):122-31.

28. Fathi R, Samadi MS, Qotbi AAA, Seidavi A, Marin ALM. Effects of feed supplementation with increasing levels of organic acids on growth performance, carcass traits, gut microbiota and pH, plasma metabolites, and immune response of broilers. Anim Sci Pap Rep. 2016;34(2):195-206.

29. Mohammadi Gheisar M, Hosseindoust A, Kim IH. Evaluating the effect of microencapsulated blends of organic acids and essential oils in broiler chickens diet. J Appl Poult Res. 2015;24(4):511-9.

30. Swaggerty CL, Arsenault RJ, Johnson C, Piva A, Grilli E. Dietary supplementation with a microencapsulated blend of organic acids and botanicals alters the kinome in the ileum and jejunum of Gallus gallus. PLoS One. 2020;15(7):e0236950.

31. Grilli $E$, Tugnoli B, Formigoni A, Massi P, Fantinati $P$, Tosi $G$, et al. Microencapsulated sorbic acid and nature-identical compounds reduced Salmonella Hadar and Salmonella Enteritidis colonization in experimentally infected chickens. Poult Sci. 2011;90(8):1676-82.

32. Grilli E, Vitari F, Domeneghini C, Palmonari A, Tosi G, Fantinati P, et al. Development of a feed additive to reduce caecal campylobacter jejuni in broilers at slaughter age: from in vitro to in vivo, a proof of concept. J Appl Microbiol. 2013;114(2):308-17.
33. Grilli E, Tugnoli B, Passey JL, Stahl CH, Piva A, Moeser AJ. Impact of dietary organic acids and botanicals on intestinal integrity and inflammation in weaned pigs. BMC Vet Res. 2015;11:96.

34. Swaggerty $\mathrm{CL}, \mathrm{He} \mathrm{H}$, Genovese KJ, Callaway TR, Kogut MH, Piva A, et al. A microencapsulated feed additive containing organic acids, thymol, and vanillin increases in vitro functional activity of peripheral blood leukocytes from broiler chicks. Poult Sci. 2020;99(7):3428-36.

35. Pham VH, Kan L, Huang J, Geng Y, Zhen W, Guo Y, et al. Dietary encapsulated essential oils and organic acids mixture improves gut health in broiler chickens challenged with necrotic enteritis. J Anim Sci Biotechnol. 2020;11:18.

36. Citalan-Madrid AF, Garcia-Ponce A, Vargas-Robles H, Betanzos A, Schnoor M. Small GTPases of the Ras superfamily regulate intestinal epithelial homeostasis and barrier function via common and unique mechanisms. Tissue Barriers. 2013;1(5):e26938.

37. Abudabos AM, Alyemni AH, Dafalla YM, Khan RU. Effect of organic acid blend and Bacillus subtilis alone or in combination on growth traits, blood biochemical and antioxidant status in broilers exposed to Salmonella typhimurium challenge during the starter phase. J Appl Anim Res. 2017; 45(1):538-42.

38. Nosrati M, Javandel F, Camacho LM, Khusro A, Cipriano M, Seidavi A, et al. The effects of antibiotic, probiotic, organic acid, vitamin C, and Echinacea purpurea extract on performance, carcass characteristics, blood chemistry, microbiota, and immunity of broiler chickens. J Appl Poult Res. 2017;26(2):295-306.

39. Faith JJ, Guruge JL, Charbonneau M, Subramanian S, Seedorf H, Goodman $\mathrm{AL}$, et al. The long-term stability of the human gut microbiota. Science. 2013;341(6141):1237439.

40. Subramanian S, Blanton LV, Frese SA, Charbonneau M, Mills DA, Gordon JI. Cultivating healthy growth and nutrition through the gut microbiota. Cell. 2015:161(1):36-48

41. Li H, Qi Y, Jasper H. Preventing age-related decline of gut compartmentalization limits microbiota dysbiosis and extends lifespan. Cell Host Microbe. 2016;19(2):240-53.

42. Svihus B. Function of the digestive system. J Appl Poult Res. 2014;23:306-14.

43. Zimonja O, Svihus B. Effects of processing of wheat or oat starch on technical pellet quality and nutritional value for broilers. Anim Feed Sci Technol. 2009;149:287-97.

44. Pan D, Yu Z. Intestinal microbiome of poultry and its interaction with host and diet. Gut Microbes. 2014;5(1):108-19.

45. Yamauchi K, Tarachai P. Changes in intestinal villi, cell area and intracellular autophagic vacuoles related to intestinal function in chickens. Br Poult Sci. 2000;41(4):416-23.

46. Metzler-Zebeli BU, Magowan E, Hollmann M, Ball MEE, Molnar A, Witter K, et al. Differences in intestinal size, structure, and function contributing to feed efficiency in broiler chickens reared at geographically distant locations. Poult Sci. 2018;97(2):578-91.

47. Hasan A, Adem Y. Influence of dietary thymol and carvacrol preparation and/ or an organic acid blend on growth performance, digestive organs and intestinal microbiota of broiler chickens. Afr J Microbiol Res. 2011;5(8):979-84.

48. Saeed M, Yatao X, Tiantian Z, Qian R, Chao S. 16S ribosomal RNA sequencing reveals a modulation of intestinal microbiome and immune response by dietary L-theanine supplementation in broiler chickens. Poult Sci. 2019;98(2):842-54

49. Wlodarska M, Willing BP, Bravo DM, Finlay BB. Phytonutrient diet supplementation promotes beneficial clostridia species and intestinal mucus secretion resulting in protection against enteric infection. Sci Rep. 2015:5:9253.

50. Abudabos AM, Alyemni AH, Dafalla YM, Khan RU. The effects of phytogenetics on growth traits, blood biochemical and intestinal histology in broiler chickens exposed to Clostridium perfringens challenge. J Appl Anim Res. 2018;46:691-5.

51. Maki JJ, Klima CL, Sylte MJ, Looft T. The microbial pecking order: Utilization of intestinal microbiota for poultry health. Microorganisms. 2019;7(10):376.

52. Vandeputte D, Kathagen G, D'Hoe K, Vieira-Silva S, Valles-Colomer M, Sabino $J$, et al. Quantitative microbiome profiling links gut community variation to microbial load. Nature. 2017;551(7681):507-11.

53. Yeoman CJ, White BA. Gastrointestinal tract microbiota and probiotics in production animals. Annu Rev Anim Biosci. 2014;2:469-86.

54. Bratburd JR, Keller C, Vivas E, Gemperline E, Li L, Rey FE, et al. Gut microbial and metabolic responses to Salmonella enterica Serovar Typhimurium and Candida albicans. mBio. 2018;9(6):e02032-18. 
55. Rivera-Chavez F, Baumler AJ. The pyromaniac inside you: Salmonella metabolism in the host gut. Annu Rev Microbiol. 2015;69:31-48.

56. Rehman T. Role of the gut microbiota in age-related chronic inflammation. Endocr Metabo Immune Disord Drug Targets. 2012:12:361-7.

57. National Research Council. Guide for the Care and Use of Laboratory Animals. 8th ed. Washington DC: Nat Acad Press; 2011.

58. National Research Council. Nutrient requirements of poultry. 9th ed. Washington DC: Nat Acad Press; 1994. p. 19-34.

59. Feye KM, Rubinelli PM, Chaney WE, Pavlidis HO, Kogut MH, Ricke SC. The preliminary development of an in vitro poultry cecal culture model to evaluate the effects of original XPC(TM) for the reduction of Campylobacter jejuni and its potential effects on the microbiota. Front Microbiol. 2019;10: 3062.

60. Kozich JJ, Westcott SL, Baxter NT, Highlander SK, Schloss PD. Development of a dual-index sequencing strategy and curation pipeline for analyzing amplicon sequence data on the MiSeq Illumina sequencing platform. Appl Environ Microbiol. 2013;79(17):5112-20.

61. Illumina. https://login.llumina.com/platform-services-manager/? rURL= https://basespace.illumina.com\&clientld=basespace\&clientVars=aHROcHM6 Ly9iYXNIc3BhY2UuaWxsdW1 pbmEuY29tL2Rhc2hib2FyZA\&redirectMethod= GET\#/. Accessed 20 Aug 2020.

62. Bolyen E, Rideout JR, Dillon MR, Bokulich NA, Abnet CC, Al-Ghalith GA, et al. Reproducible, interactive, scalable and extensible microbiome data science using QIIME 2. Nat Biotechnol. 2019;37(8):852-7.

63. Callahan BJ, McMurdie PJ, Rosen MJ, Han AW, Johnson AJ, Holmes SP. DADA2: high-resolution sample inference from Illumina amplicon data. Nat Meth. 2016;13(7):581-3.

64. Mandal S, Van Treuren W, White RA, Eggesbo M, Knight R, Peddada SD. Analysis of composition of microbiomes: a novel method for studying microbial composition. Microb Ecol Health Dis. 2015;26:27663.

65. Galaxy Community Hub. https://galaxyproject.org. Accessed 20 Aug 2020.

66. Segata N, Izard J, Waldron L, Gevers D, Miropolsky L, Garrett WS, et al. Metagenomic biomarker discovery and explanation. Genome Biol. 2011; 12(6):R60

\section{Publisher's Note}

Springer Nature remains neutral with regard to jurisdictional claims in published maps and institutional affiliations.

Ready to submit your research? Choose BMC and benefit from:

- fast, convenient online submission

- thorough peer review by experienced researchers in your field

- rapid publication on acceptance

- support for research data, including large and complex data types

- gold Open Access which fosters wider collaboration and increased citations

- maximum visibility for your research: over $100 \mathrm{M}$ website views per year

At $\mathrm{BMC}$, research is always in progress.

Learn more biomedcentral.com/submissions 